Imaging, DTI) genauer an. Insgesamt analysierten sie damit die Gehirne von 17 bisher nicht behandelten Patienten mit ausgeprägter Schlafapnoe sowie von 15 gleichaltrigen gesunden Probanden (Alter 30 bis 55 Jahre).

Die Messungen erfolgten zu Beginn der Studie sowie drei und zwölf Monate nach Beginn einer CPAP-Therapie. Ausgiebig prüften die Forscher mit einer neuropsychologischen Testbatterie auch die kognitiven Fähigkeiten der Teilnehmer.

\section{Kognitionsdefizite durch Läsionen der Faserbahnen?}

Wie erwartet, zeigten die SchlafapnoePatienten gegenüber den gesunden Teilnehmern ausgeprägte Defizite in den meisten kognitiven Bereichen. Zugleich deuten die DTI-Aufnahmen auf eine Schädigung wichtiger Faserverbindungen. So beobachtete das Team um FeriniStrambi eine reduzierte fraktionelle Anisotropie und eine verminderte Diffusion in einigen Hirnarealen. Eine geringe fraktionelle Anisotropie deutet auf mikrostrukturelle Schäden der betroffenen Fasern, die verminderte Diffusion kann wiederum Folge zytotoxisch-ödematöser Prozesse sein, bei denen die Axone und Zellkörper der Nervenzellen anschwellen, dadurch den extrazellulären
Raum verringern und die Wasserdiffusion erschweren. Betroffen von den Veränderungen waren hauptsächlich Faserverbindungen im Corpus callosum, Fornix, Cingulum sowie kurze und lange Assoziationsbahnen.

Die gute Nachricht: Wie die Forscher nun feststellten, waren auch diese Veränderungen komplett reversibel. Allerdings benötigte dieser Prozess deutlich mehr Zeit als die Rückbildung der Atrophie der grauen Substanz. Erst nach einem Jahr CPAP-Therapie waren die Werte der fraktionellen Anisotropie und der mittleren Diffusion weitgehend normal. In dieser Zeit verschwanden auch fast alle kognitiven Defizite der Schlafapnoe-Patienten, sogar bei den exekutiven Funktionen war eine Verbesserung $\mathrm{zu}$ verzeichnen, allerdings nicht im Stroop-Test und auch das Langzeitgedächtnis hatte noch nicht das Niveau der gesunden Probanden erreicht.

Insgesamt, so Ferini-Strambi, scheint die Verbesserung der kognitiven Funktionen eher mit einer Reduktion struktureller Schäden der weißen Substanz als mit der Regeneration der grauen Substanz zu korrelieren. Es bestehe daher die Hoffnung, dass eine konsequente und langfristige CPAP-Therapie die Schäden komplett rückgängig mache - allerdings nur bei konsequenter Therapie: In einer Studie mit CPAP waren die kognitiven Defizite nach drei Monaten zu $70 \%$ behoben - jedoch nur bei den Patienten, die im Schnitt mehr als sechs Stunden pro Nacht unter Beatmung schliefen. Nutzten sie die Therapie weniger als zwei Stunden pro Nacht, kam es kaum zu Verbesserungen. Die schlechte Compliance sei daher vermutlich auch ein Grund, weshalb es in Studien mit CPAP zu keiner kompletten Normalisierung der kognitiven Funktion kommt.

Für Ferini-Strambi lohnt es sich, auch mit Blick auf das erhöhte Risiko für kognitive Beeinträchtigung und Demenz, bei Schlafapnoe-Patienten die CPAPTherapie $\mathrm{zu}$ forcieren. Zwar ist bisher nicht belegt, dass die Therapie eine Demenz verhindern kann, die strukturellen Schäden, die sich per DTI-Bildgebung bei unbehandelten Patienten nachweisen lassen, bekräftigen jedoch die Hypothese, dass zerebrale Perfusionsstörungen bei Schlafapnoe zu neuronalen Schädigungen führen, die eine Demenz begünstigen können. Deshalb sei die CPAP auf jeden Fall einen Versuch wert.

Thomas Müller

Symposium "Sleep Disorders", 23. Meeting der European Neurological Society (ENS), Barcelona, 10.6.2013

\title{
Nächtliche Beatmung stoppt Migräne bei Schlafapnoe
}

\author{
Schlafapnoe und dazu noch Migräne - das ist keine seltene Kombination. Oft ist der nächtliche \\ Sauerstoffmangel sogar Auslöser der Kopfschmerzen.
}

twa jeder fünfte bis jeder vierte Pati-
Migrt mit Schlafapnoe berichtet über
nach dem Aufwachen. Als Trigger ver-
mutet man nächtliche Hypoxien, erklär-
te Hildegard Hidalgo von der Kamillus-
Klinik in Asbach. Daher müsste eine
nächtliche Beatmung mit CPAP eigent-
lich auch die Kopfschmerzen lindern.
Dies überprüfte das Team um Hidalgo
an 41 Männern und Frauen (Durch-
schnittsalter 50 Jahre), die unter
Schlafapnoe und Migräne litten. Vor der
CPAP-Therapie hatten die Patienten im
Schnitt fünf Migräneattacken pro Mo-
nat, der Apnoe-Hypopnoe-Index (AHI) lag im Mittel bei 27 Punkten. Nach einem Jahr waren bei den Patienten mit CPAP die Migräneattacken praktisch komplett verschwunden: Im Schnitt hatten die Betroffenen nur noch 0,2 Attacken pro Monat, nach zwei Jahren waren es nur noch 0,1 pro Monat. Die verbliebenen Attacken waren weniger schmerzhaft: Auf einer Intensitätsskala war der Wert um etwa $80 \%$ zurückgegangen, auch der Konsum von Schmerzmitteln war entsprechend deutlich reduziert. Der AHI-Wert lag jetzt nur noch bei 2,2 Punkten, die Sauerstoffsättigung war besser und die Patienten verbrachten mehr Zeit im Tiefschlaf.
Insgesamt, so Hidalgo, scheint die CPAP-Therapie sehr wirkungsvoll eine Migräne bei Schlafapnoe zu stoppen. Bei Migräne-Patienten mit Risikofaktoren für Schlafapnoe wie Übergewicht sollte daher geprüft werden, ob eine bisher nicht therapierte Schlafapnoe vorliegt. Kurz gesagt: Dicke Migräne-Patienten nicht gleich mit Triptanen behandeln, sondern erst einmal fragen, ob sie nachts laut schnarchen!

Thomas Müller

Symposium "Sleep Disorders", 23. Meeting der European Neurological Society (ENS), Barcelona, 10.6.2013 